\title{
Implications of the Unexpected Persistance of Human Rhinovirus/Enterovirus during the COVID-19 Pandemic in Canada
}

\author{
David Champredon ${ }^{1}$, Christina Bancej ${ }^{1}$, Liza Lee $^{1}$, and Steven Buckrell ${ }^{1}$ \\ ${ }^{1}$ Public Health Agency of Canada
}

October 17, 2021

\begin{abstract}
Stringent public health measures imposed across Canada to control the COVID-19 pandemic have nearly suppressed most seasonal respiratory viruses, with the notable exception of human rhinovirus/enterovirus (hRV/EV). Thanks to this unexpected persistence, we highlight that $\mathrm{hRV} / \mathrm{EV}$ could serve as a sentinel for levels of contact rate in populations to inform on the efficiency $\neg$, or the need of, public health measures to control the subsequent COVID-19 epidemic, but also for future epidemics from other seasonal or emerging respiratory pathogens.

David Champredon ${ }^{1, *}$, Christina Bancej ${ }^{2}$, Liza Lee $^{2}$, Steven Buckrell ${ }^{2}$

${ }^{1}$ Public Health Agency of Canada, National Microbiology Laboratory. Public Health Risk Sciences Division. Guelph, Ontario.

${ }^{2}$ Public Health Agency of Canada, Centre for Immunization and Respiratory Infectious Disease. Surveillance and Epidemiology Division. Ottawa, Ontario.

* Corresponding author: david.champredon@canada.ca

Author contributions:

David Champredon: Conceptualization, Writing - Original Draft Preparation, Formal Analysis.

Christina Bancej: Conceptualization, Writing - Review \& Editing.

Liza Lee: Data Curation, Writing - Review \& Editing.

Steven Buckrell: Data Curation.

Conflict of interest: None.

Funding statement: None.

Data availability statement: all data is publicly available

Ethics approval statement: not applicable.
\end{abstract}

Keywords : Rhinovirus, SARS-CoV-2 / COVID-19, respiratory viruses surveillance, contact rate.

\section{Summary}

Stringent public health measures imposed across Canada to control the COVID-19 pandemic have nearly suppressed most seasonal respiratory viruses, with the notable exception of human rhinovirus/enterovirus 
(hRV/EV). Thanks to this unexpected persistence, we highlight that hRV/EV could serve as a sentinel for levels of contact rate in populations to inform on the efficiency, or the need of, public health measures to control the subsequent COVID-19 epidemic, but also for future epidemics from other seasonal or emerging respiratory pathogens.

\section{Main Text (Short Communication)}

The 2019-20 seasonal respiratory virus activity in Canada followed a typical trajectory until March 2020, when a series of stringent public health measures (PHM) aimed at controlling cases of COVID-19 were imposed in many jurisdictions across Canada. The PHMs essentially reduced the effective transmission rate of SARS-CoV-2, but also of other pathogens transmitted by the respiratory route. Hence, in Canada and other countries, the prevalence of most seasonal respiratory viruses (including influenza and respiratory syncytial virus (RSV)) ended abruptly in mid-March and were virtually suppressed or well below historical levels throughout the 2020-21 season in Canada (Figure S1). A notable exception were human rhinovirus/enterovirus (hRV/EV) infections which persisted through repeated stringent PHMs retaining, to some extent, their typical seasonal patterns in many Canadian jurisdictions.

The causes of hRV/EV persistence are not fully understood yet. The fact that hRV/EV are non-enveloped may help sustain transmission thanks to their prolonged survival on surfaces . Masks may be less effective at stopping droplets and aerosols transporting $\mathrm{hRV} / \mathrm{EV}$. Another possibility may be that the population groups that drive $\mathrm{hRV} / \mathrm{EV}$ transmission (typically young children) did not experience a contact rate reduction as effective as the groups driving SARS-CoV-2 transmission when social distancing and other PHMs were in place. Alternatively, hRV/EV transmissibility to and among children may be similar to or higher than that of SARS-CoV-2 in the pediatric population. Finally, the baseline prevalence of hRV/EV may be significantly higher than other respiratory infections (it also shows typical summertime persistence), allowing hRV/EV to rebound more easily when PHMs ease.

The persistence of hRV/EV highlights a new and unsuspected role for this family of viruses as a sentinel for the transmission rate for respiratory pathogens. Figure 1 suggests that, with the hindsight of 18 months of observations for both hRV/EV and SARS-CoV-2 infections in Canada, hRV/EV could have been used as a gauge for PHMs effectiveness as well as early warning for SARS-CoV-2 resurgences. Indeed, in four large provinces, where the signal is not too hampered by observation noise (hRV/EV is not reported by the province of Quebec, the second most populous Canadian province), the positivity rate of hRV/EV approximately mirrors, and often precedes by several weeks, the positivity rate for SARS-CoV-2 during the period when the vaccination coverage was null or low. Although the data to disentangle the intrinsic seasonality of $\mathrm{hRV} / \mathrm{EV}$ infections and the effect of COVID-19 PHMs are not available, it is conceivable that PHMs did impact $\mathrm{hRV} / \mathrm{EV}$ detection rates, making them a potential early monitor of the efficacy of PHMs. When COVID-19 vaccine coverage reached a substantial proportion of the population (starting in May 2021), a statistical analysis between SARS-CoV-2 and the lagged hRV/EV positivity rates suggests a decoupling of their incidence (Supplementary Material); the latter are not vaccine-preventable and thus followed their typical seasonality and intensity beyond May 2021.

The COVID-19 pandemic has provided a real-life experiment on how stringent PHMs on social distancing and enhanced personal protective measures (e.g., hand hygiene, respiratory etiquette, and face masks) impacted the circulation of seasonal respiratory viruses. Notably, it highlighted the unique role for hRV/EV incidence to gauge the contact rate of a population (most likely its younger age groups, where hRV/EV symptomatic infections may be more prevalent). Direct measurements of contact rates through population surveys (for example ) are valuable but not sufficiently timely for surveillance, near-term forecasting and thus for early warning of resurgence/re-emergence potential for respiratory infectious diseases. Testing volumes for hRV/EV in Canada during the 2019-20 and 2020-21 seasons were higher than pre-pandemic levels (Figure S4), suggesting potential different demographics tested during non-pandemic seasons. 
Our observations support the continuation for routine acute respiratory infections

surveillance that includes hRV/EV, and encourages enhancements (e.g., sampling and reporting across all ages and spectrum of illness). The current COVID-19 pandemic has shown that monitoring the incidence of $\mathrm{hRV} / \mathrm{EV}$ may have been undervalued so far: it could be useful for the subsequent SARS-CoV-2 epidemic activity, and for the longer term, potentially providing early warnings for other seasonal respiratory illnesses (notably influenza and RSV) as well as the transmission intensity at the time of introduction of another emerging respiratory pathogen in the monitored population.

\section{Hosted file}

Figure-1-caption.docx available at https://authorea.com/users/441493/articles/541976implications-of-the-unexpected-persistance-of-human-rhinovirus-enterovirus-during-thecovid-19-pandemic-in-canada 